\title{
Action conjointe et connaissances professionnelles de l'enseignant
}

Joint action theory and teacher's professional knowledge

David Cross

\section{(2) OpenEdition}

\section{Journals}

\section{Édition électronique}

URL : http://journals.openedition.org/educationdidactique/850

DOI : $10.4000 /$ educationdidactique.850

ISBN : 978-2-7535-1625-0

ISSN : $2111-4838$

\section{Éditeur}

Presses universitaires de Rennes

\section{Édition imprimée}

Date de publication : 15 décembre 2010

Pagination : $39-60$

ISBN : 978-2-7535-1301-3

ISSN : 1956-3485

\section{Référence électronique}

David Cross, "Action conjointe et connaissances professionnelles de l'enseignant », Éducation et didactique [En ligne], 4-3 | Décembre 2010, mis en ligne le 10 décembre 2012, consulté le 09 décembre 2020. URL : http://journals.openedition.org/educationdidactique/850 ; DOI : https://doi.org/10.4000/ educationdidactique. 850 


\title{
ACTION CONJOINTE ET CONNAISSANCES PROFESSIONNELLES DE L'ENSEIGNANT
}

\author{
David Cross (UMR ICAR, Université Lyon 2)
}

\begin{abstract}
Résumé : Lorsque l'on s'intéresse à la formation des enseignants ou à l'étude des pratiques de classe, les connaissances professionnelles des enseignants occupent une place privilégiée. Ces connaissances de l'enseignant sont donc au cœur d'un certain nombre d'études en didactique (Abell, 2007). Ces études reposent sur un certains nombres d'hypothèses, souvent implicites, sur le lien entre ce que les enseignants disent de leurs pratiques et ce qu'ils font effectivement dans leur classe. Or ce lien est loin d'être clair, et ce sont principalement les connaissances sur l'action qui sont étudiées par cette approche. Afin de dépasser cette limitation et d'étudier également les connaissances dans l'action nous proposons une articulation théorique entre la TACD et des catégories de connaissances professionnelles mises au jour par des travaux d'un autre courant scientifique (Pedagogical Content Knowledge) ainsi qu'une méthode d'analyse de ces connaissances en lien avec la théorie de l'action conjointe.

Mots Clés : Théorie de l'Action Conjointe en didactique (TACD), Pedagogical Content Knowledge (PCK), Connaissances professionnelles des enseignants, Didactique de la chimie.
\end{abstract}

\section{De l'intérêt d'étudier les Pedagogical Content Knowledge à l'aide de la TACD}

Le cadre théorique des Pedagogical Content Knowledge (PCK) a été développé dans une perspective de formation des enseignants. Ce cadre consiste principalement en une catégorisation des connaissances des enseignants, catégorisation qui est faite a priori et en cherchant à répondre à la question: de quelles connaissances a besoin un enseignant pour enseigner un certain contenu de savoir? Nous estimons que cette approche ne contient pas de théorisation solide c'est-à-dire d'un système de concepts à la clef. L'hypothèse à la base de ce cadrage, plus ou moins explicite, est que les connaissances de l'enseignant (que celles-ci soient disciplinaires ou sur l'enseignement) influencent directement ses pratiques (et donc l'action de l'enseignant hors classe et dans la classe).

La question des connaissances nécessaires pour enseigner un contenu de savoir est cruciale lorsqu'il s'agit de former des enseignants et de comprendre le lien entre ce qu'ils savent et leur agir en classe et mérite ainsi une attention toute particulière de la part de la communauté des chercheurs en éducation.

La plupart des études sur les PCK ont cherché à identifier les connaissances des enseignants à partir de ce qu'ils disent sur leurs connaissances et leur pratique, il s'agit donc de reconstruire des connaissances sur l'action. En effet les méthodologies mises en œuvre dans ces études sont basées sur des questionnaires et des interviews. Ces études ne théorisent pas le statut de ce qui est dit (en tant que discours adressé, sous couvert d'une institution de recherche donnée, dans un dispositif spécifié, etc.): elles supposent donc un lien relativement direct entre ce qu'un enseignant dit de ses connaissances et de sa pratique et ce qu'il fait dans sa pratique. Or d'après plusieurs chercheurs ce lien est loin d'être clair (Kagan, 1992; Carter, 1993). Il existe bien sûr une certaine cohérence entre ce qui est dit et ce qui est fait parles enseignants, ainsi l'étude de Park et Oliver (2008), entre autres, montre que les PCK mises à jour dans le discours de l'enseignant sur sa pratique font écho à certaines de ses actions dans sa pratique. Plusieurs autres études, par exemple celle de Rollnick et al. (2008) ont ainsi cherché à documenter la mise en œuvre de PCK à partir de ce que l'enseignant dit sur sa pratique dans la classe, notamment à l'aide de données vidéos de situations de classe. Mais la méthodologie employée (partir de ce qui est dit par l'enseignant pour repérer la mise en œuvre de PCK dans les données vidéo), ne permet de documenter que les PCK que l'enseignant explicite dans son discours. De plus le lien entre ce qui est dit et ce qui est fait n'est pas discuté, et la méthode qui permet de repérer l'action correspondant à la mise en œuvre d'une PCK reste du domaine de l'implicite. 
Les PCK offrent une catégorisation intéressante pour étudier à la fois les pratiques enseignantes et réfléchir sur la formation des enseignants. En effet plus de vingt ans de travaux sur ce sujet ont permis d'obtenir un modèle (constitué de catégories de connaissances) faisant globalement consensus au sein des formateurs d'enseignants. Cette constance dans les efforts sur la recherche portant sur les PCK, montre que ce concept ainsi que les catégories de connaissances ont un vrai potentiel heuristique.

Une façon de contourner le problème du lien entre les connaissances étudiées à partir de ce que les enseignants disent de leur pratique et les connaissances effectivement mises en ouvre dans leur pratique est d'étudier ces connaissances directement à partir de la pratique, et de reconstruire ainsi des connaissances dans l'action. Il ne s'agit plus de repérer a priori la mise en œuvre de connaissances dans une pratique professionnelle, mais de faire émerger celles-ci à partir de l'analyse des actions du professionnel. Nous proposons donc une approche nouvelle en ce qui concerne l'étude des connaissances professionnelles. En partant de l'observation et de l'analyse de la pratique de classe nous cherchons à reconstruire des connaissances mises en œuvre. La pratique de classe sera analysée grâce à la théorie de l'action conjointe en didactique (TACD).

Notre étude cherche donc à mettre en lumière les connaissances professionnelles mises en œuvre par l'enseignant dans sa classe et ceci dans deux buts:

- Pouvoir décrire la pratique de classe du point de vue des connaissances de l'enseignant;

- Pouvoir construire des savoirs professionnels ${ }^{1}$ à partir de connaissances effectivement mises en œuvre. Ces savoirs professionnels présentant un potentiel fort dans le cadre de la formation des enseignants. La construction de savoirs professionnels soulève de nombreuses questions, nous nous limiterons donc, dans un premier temps, à la mise en lumière des connaissances mises en oeuvre.

Notre but est donc d'étudier les connaissances professionnelles à partir de l'action de l'enseignant dans sa classe. La TACD offre un cadre interprétatif de cette action, sans parler explicitement de connaissances professionnelles. Il nous faut donc articuler la
TACD au cadre des PCK, pour pouvoir reconstruire des connaissances à partir des observables de l'action. Nous verrons également si le. modèle des connaissances professionnelles des enseignants développé par l'approche en termes de PCK reste pertinent dans le cadre de la TACD.

\section{Cadre théorique}

\section{Les Pedagogical Content Knowledge}

Shulman $(1986,1987)$ est parti du constat que les investigations portant sur les connaissances des enseignants portaient soit sur les connaissances disciplinaires, soit sur les connaissances pédagogiques. Pour Shulman, ces recherches ne permettaient pas de répondre à une question essentielle: quelles connaissances sont nécessaires pour enseigner la chimie? l'histoire? etc. Il a donc proposé un modèle des connaissances des enseignants permettant de répondre à cette question.

Ce modèle consiste à distinguer différents types de connaissances de l'enseignant et de les catégoriser. Dans ce modèle, les connaissances des enseignants peuvent appartenir à quatre catégories en relation entre elles:

- Les connaissances du contenu disciplinaire: il s'agit des connaissances que l'enseignant est chargé d'enseigner aux élèves, mais ses connaissances disciplinaires ne se limitent pas à celles qu'il doit enseigner. Les connaissances du contenu disciplinaire peuvent être de deux natures: elles peuvent porter sur l'organisation des concepts, faits, principes et théories ou bien sur les règles régissant les preuves utilisées pour généraliser et justifier les connaissances produites par la discipline (Abell, 2007).

- Les connaissances pédagogiques font référence aux connaissances générales de l'enseignant à propos de l'enseignement, sans être liées à un contenu disciplinaire. Par exemple de savoir qu'il faut varier les activités pour maintenir l'intérêt des élèves.

- Les connaissances sur le contexte regroupent les connaissances que possède l'enseignant à propos de l'origine de ses élèves (classe sociale etc.), du type 
d'établissement dans lequel il enseigne etc. et qu'il prend en compte dans son enseignement.

- Les connaissances pédagogiques liées au contenu (Pedagogical Content Knowledge, PCK) : ce sont les connaissances que développe un enseignant pour aider les élèves à comprendre et apprendre un contenu.

Initialement le concept de PCK a été introduit par Shulman $(1986$; 1987) comme une forme particulière de connaissances du contenu, transformées dans le but d'enseigner. Shulman a décrit cette transformation comme survenant lorsqu'un enseignant réfléchit de manière critique sur un contenu et l'interprète, trouve des manières de le présenter (analogies, métaphores, problèmes, expériences...) et adapte son enseignement aux capacités et aux idées des élèves. Pour Shulman, les PCK peuvent se voir comme un amalgame entre des connaissances pédagogiques et des connaissances disciplinaires.
Hashweh (2005) conceptualise les PCK comme une collection d'unités plus petites appelées Teacher Pedagogical Constructions (TPC, Construction Pédagogique de l'Enseignant). Ces TPC sont en interaction avec les autres types de connaissances (connaissances sur le contenu, le contexte et pédagogique dans le modèle de Grossman).

Les TPC peuvent être catégorisées en fonction de ce sur quoi elles portent. Nous reprenons, à cette fin, la catégorisation de Magnusson, Krajcik, et Borko, (1999). Les composantes sont les suivantes: connaissances sur les difficultés des élèves, connaissances sur les stratégies d'enseignement, connaissances sur l'évaluation, connaissances sur le contexte. Chacune de ces composantes est reliée à la composante « buts et valeurs de l'enseignement des sciences » (figure 1). Cette dernière chapeaute ainsi chacune des autres composantes. Ces cinq catégories de connaissances sont elles-mêmes constituées de sous-catégories.

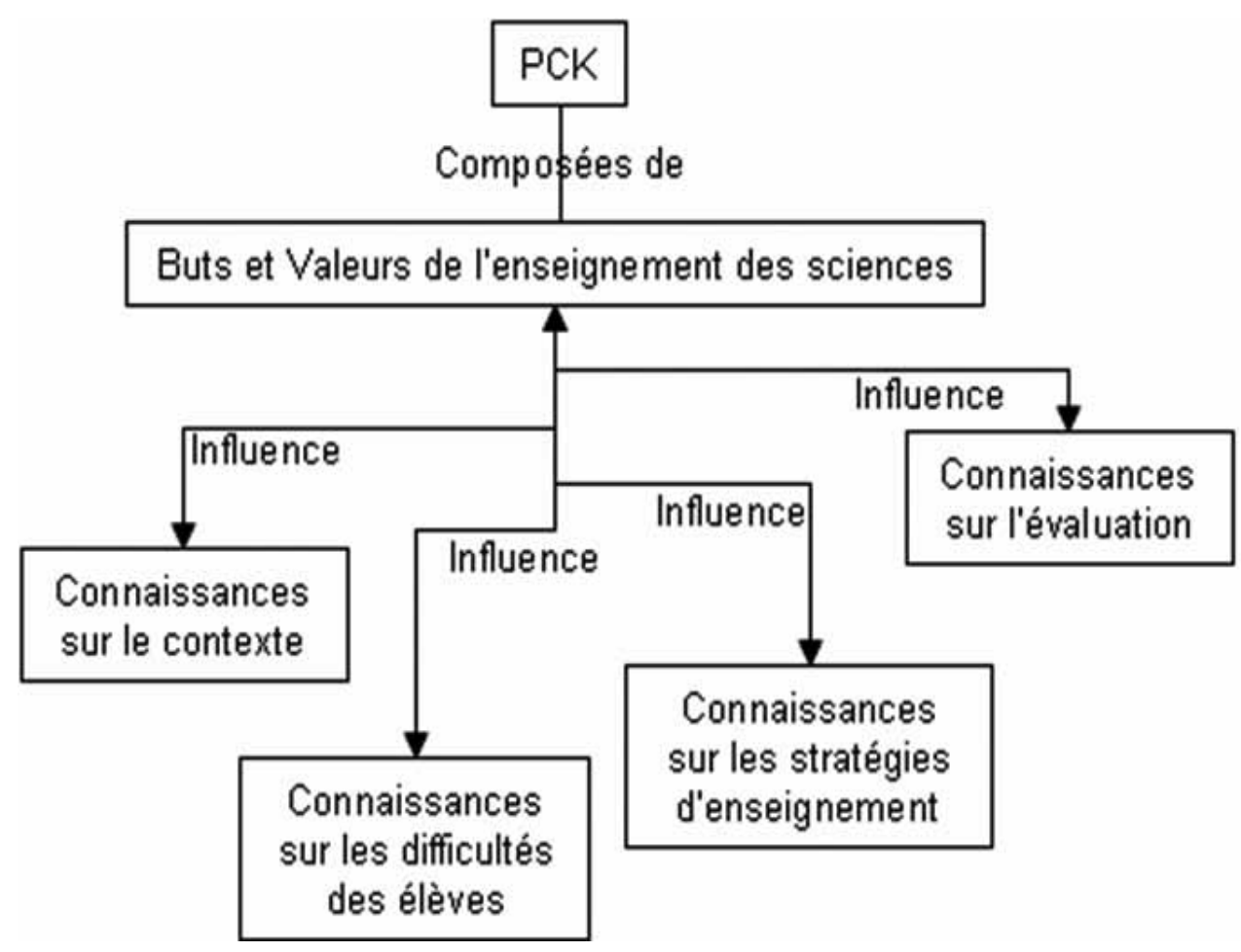

Figure 1: Modèle développé par Magnusson et al. (1999) sur les différentes composantes des PCK. 


\section{Connaissances sur les difficultés des élèves}

La première composante que nous allons envisager concerne les connaissances que les enseignants ont à propos des difficultés des élèves. D'après Magnusson, Krajcik, et Borko, (1999), cette catégorie contient deux sous-catégories: les connaissances sur les requis nécessaires pour apprendre des concepts scientifiques spécifiques et les connaissances sur les parties difficiles d'un contenu pour les élèves.

\section{Connaissances sur les requis}

Cette sous-catégorie comprend les connaissances de l'enseignant à propos des connaissances que les élèves doivent avoir pour apprendre un contenu scientifique.

Par exemple, si le but d'un enseignant est d'illustrer le concept de température par des mesures expérimentales réalisées par les élèves, l'enseignant doit anticiper les connaissances dont les élèves vont avoir besoin (par exemple mesurer la température et interpréter les résultats). Savoir comment leur faire acquérir ces connaissances si les élèves ne les possèdent pas fait aussi partie des connaissances rattachées à cette composante.

Citons également dans cette sous-catégorie les connaissances de l'enseignant vis-à-vis des différences entre élèves, face à l'apprentissage d'un contenu spécifique, en fonction de leurs capacités. Ainsi, lors de l'enseignement d'un phénomène à l'échelle moléculaire en chimie, certains élèves vont être capables de raisonner à partir d'une formule chimique, d'autres auront besoin d'un modèle en trois dimensions, etc.

\section{Connaissances sur les parties difficiles}

Cette sous-catégorie fait référence aux connaissances des enseignants à propos des concepts scientifiques ou des sujets que les élèves trouvent difficiles à apprendre. De multiples raisons rendent compte des difficultés des élèves pour apprendre certaines choses. Par exemple on peut noter que le niveau d'abstraction de certains sujets est trop élevé et/ou la connexion avec le monde quotidien des élèves est trop faible.
D'autres sujets sont difficiles parce que l'enseignement centre l'activité des élèves sur la résolution de problèmes et que certains d'entre eux éprouvent des difficultés à en planifier la résolution. Enfin, une troisième source de difficulté vient du fait que l'apprentissage du sujet à enseigner nécessite des connaissances en contradiction avec les connaissances naives des élèves (issues de l'expérience quotidienne des élèves).

\section{Connaissances sur les stratégies d'enseignement}

Les stratégies d'enseignement correspondent à des connaissances de l'enseignant sur ses manières de présenter un savoir (métaphore, analogie, modèle, exemples...) d'une part, et sur ses connaissances liées aux activités qui peuvent aider les élèves à comprendre un contenu d'autre part.

\section{Connaissances sur les manières de présenter un savoir}

Illustrons cette catégorie à partir des analogies représentant le courant électrique. Il en existe plusieurs: de l'eau circulant dans un circuit fermé et mue par une pompe, une chaîne de vélo ou encore une foule se déplaçant. Chacune présente des avantages et des inconvénients. Par exemple, l'analogie de l'eau circulant dans un circuit fermé risque de renforcer chez l'élève une vision de l'électricité basée sur le modèle "source-receveur », et implique que les'électrons se déplacent dans une même direction. Lanalogie de la chaîne de vélo implique également que les électrons se déplacent dans la même direction, mais par contre évite de renforcer le modèle source-receveur. L'analogie de la foule se déplaçant permet de distinguer le mouvement d'une personne et celui de la foule illustrant ainsi que le flux de charges dans un circuit électrique a une direction distincte du mouvement aléatoire des électrons. Savoir quel modèle utiliser est une TPC appartenant à cette composante des PCK.

\section{Connaissances sur les types de travaux}

Cette sous-catégorie fait référence aux connaissances à propos des types de travaux qui peuvent être mises en place afin d'aider les élèves à comprendre certains concepts ou relations. 


\section{Connaissances sur l'évaluation}

Cette composante comporte des sous-catégories relatives aux aspects de l'apprentissage des sciences qu'il est important d'évaluer et aux connaissances relatives à leur évaluation.

\section{Connaissances sur les aspects à évaluer}

Lapprentissage en science peut être évalué suivant différents aspects, par exemple: l'utilisation des concepts, les capacités expérimentales, la résolution de problèmes... Cette sous-catégorie de connaissances est fortement influencée par les connaissances de la composante « Buts et Raisons » de l'enseignement des sciences. Cependant, certains aspects de l'apprentissage des sciences sont plus ou moins faciles à évaluer en fonction du contenu. Ainsi, les capacités expérimentales des élèves seront plus difficiles à évaluer lors d'un enseignement portant sur la radioactivité (pour des raisons de sécurité) ou sur l'astronomie (pour des problèmes pratiques: faire observer aux élèves le système solaire en plein jour, avec quel matériel?), que dans le cadre d'un enseignement sur le circuit électrique (matériel facilement disponible, possibilité de travailler à faible puissance). La planification de l'évaluation des différents aspects de l'apprentissage des sciences en fonction du contenu le plus approprié nécessite des TPC appartenant à cette sous-catégorie.

\section{Connaissances sur les méthodes d'évaluation}

Cette sous-catégorie de la composante Connaissances sur l'évaluation correspond aux connaissances ayant trait aux façons qui peuvent être employées pour évaluer, de manière spécifique, un certain aspect de l'apprentissage des sciences par un élève. Savoir quelle méthode utiliser en fonction de l'aspect de l'apprentissage à évaluer représente une TPC de cette sous-catégorie.

\section{Connaissances sur le curriculum}

Cette composante est constituée de deux souscatégories: la connaissance du programme officiel et les connaissances relatives au matériel et objets disponibles pour l'enseignement d'un contenu.

\section{Connaissances du programme}

Cette sous catégorie regroupe les connaissances des enseignants à propos du programme officiel dans leur discipline ainsi que l'articulation de celui-ci avec le programme d'autres disciplines.

\section{Connaissances du matériel}

Nous retrouvons ici les connaissances qu'a l'enseignant sur le matériel disponible dans son lycée et qu'il peut utiliser pour son enseignement ainsi que l'ensemble des documents existant se rapportant à l'enseignement d'un contenu. Par exemple les enseignants peuvent décider de se servir d'un document mis au point par un collègue, parce qu'il estime que ce document est adapté à l'enseignement d'un contenu.

\section{Buts et valeurs de l'enseignement des sciences}

Cette composante des PCK contient les connaissances des enseignants à propos des buts et valeurs de l'enseignement des sciences à un niveau donné. Ces connaissances orientent les décisions de l'enseignant à propos de sujets tels que les objectifs à atteindre, l'utilisation du manuel scolaire ou encore l'évaluation de l'apprentissage des élèves. Cette composante chapeaute donc les autres composantes de ce modèle. Nous ajoutons à cette composante un élément qui nous semble essentiel mais qui n'apparaît pas dans le modèle de Magnusson: l'épistémologie de l'enseignant. Nous entendons par là ses connaissances sur le fonctionnement des sciences. Ce nouvel élément appartient bien à la catégorie Buts et Valeurs sur l'enseignement puisque cette épistémologie va venir influencer directement les buts qu'accorde l'enseignant à l'enseignement des sciences et les valeurs d'un tel enseignement et également fournir un fil rouge pour l'enseignant.

Magnuson et al. (1999) insistent sur le fait que ces différentes catégories de connaissances interagissent entre elles. Cet aspect du modèle nous semble être très important, surtout lorsqu'il s'agit de prendre en considération des connaissances dans l'action. La catégorie de TPC «Buts et valeurs » est présentée dans le modèle de Magnusson et al. comme une caté- 
gorie de connaissances au même titre que les autres connaissances. Or des buts et des valeurs peuvent difficilement être définies comme des connaissances stricto sensu, il s'agit en tout cas de « connaissances » se différenciant des autres types de connaissances présents dans le modèle en ce qu'elles sont certainement beaucoup moins dicibles ou beaucoup plus difficile à reconstruire par le chercheur, que ce soit à partir de du discours de l'enseignant sur sa pratique ou de l'étude de la pratique de l'enseignant.

Les TPC peuvent donc être caractérisées en fonction de ce sur quoi elles portent. Nous proposons une deuxième façon de catégoriser les TPC: en fonction du savoir enseigné auquel les TPC sont liées. En effet les TPC peuvent être distinguées selon le champ d'application, dans le cadre de l'enseignement observé, du savoir enseigné auquel elles sont liées: les TPC locales des TPC globales. Les TPC locales sont définies comme étant liées à un élément de savoir enseigné dont le champ d'application est relativement restreint. Par exemple une TPC liée à l'enseignement de la mesure du $\mathrm{pH}$ avec un pH-mètre, sera qualifiée de locale puisque cet élément de savoir est mis en œuvre principalement lorsque les élèves ont à mesurer le $\mathrm{pH}$ d'une solution à l'aide d'un pH-mètre. Les TPC globales sont quant à elles définies comme étant liées à un élément de savoir enseigné dont le champ d'application est plus grand, par exemple une TPC liée au concept de constante d'équilibre sera globale. En effet cette notion est mise en œuvre dans le cadre de l'enseignement sur les acides et les bases, l'oxydoréduction ou encore en chimie organique.

Cette conceptualisation des PCK en termes de collections d'objets plus petits permet, dans le cadre de notre étude, de contourner un problème méthodologique: les PCK sont décrites comme étant des connaissances complexes, difficiles à saisir parce que dépendantes du contexte. Il semble alors très (trop?) ambitieux de vouloir reconstruire ces connaissances à partir de l'action. Il est par contre plus raisonnable de chercher à reconstruire de petits éléments de connaissances et d'envisager les PCK comme étant une collection de ces petits éléments.

\section{Théorie de l'action conjointe en didactique}

Nous présentons dans cette partie notre point de vue sur l'action. Nous suivons Sensevy (2007) lorsque celui-ci propose de modéliser l'action didactique en termes de jeu. Nous verrons comment décrire ces jeux et comment l'intentionnalité de l'enseignant a été prise en compte.

\section{Jeu didactique}

Laction didactique peut être modélisée comme un jeu, ce qui permet de prendre en compte l'engagement affectif des acteurs ainsi que la façon dont le jeu est gagné (quelles sont les règles pour gagner, quels enjeux?). Le jeu didactique est défini ainsi par Sensevy (2007):

"On considère deux joueurs, A et B. Pour gagner au jeu, le joueur A doit produire certaines stratégies. Ces stratégies, il doit les produire de son propre mouvement, proprio motu. B accompagne A dans ce jeu. B gagne lorsque A gagne, c'est-à-dire lorsque A produit les stratégies gagnantes (raisonnablement) proprio motu. Ce dernier point représente une caractéristique marquante du jeu didactique. C'est un jeu organiquement coopératif: A ne peut prétendre gagner sans B, B ne peut prétendre gagner sans A. Seconde caractéristique majeure: $B$ possède les informations nécessaires à la production des stratégies gagnantes dont A doit témoigner la maîtrise. Toutefois, il ne peut en aucun cas les communiquer directement en $A$, ce qui irait à l'encontre de la "clause" proprio motu.

Le type de jeu pour lequel A doit produire des stratégies gagnantes (le type de jeu auquel il doit gagner) n'est bien sûr pas indifférent. C'est un jeu de savoir, qui possède entre autres particularités celle, centrale, de l'appropriation effective des stratégies: on peut toujours faire semblant de savoir quelque chose, on peut toujours mimer le savoir, mais il est des situations au sein desquelles la "supercherie" est vite démasquée.

La situation se complexifie du fait que c'est le joueur B qui est en général "juge et partie" dans le jeu didactique. Il doit en effet permettre à $A$, de façon nécessairement indirecte, de produire les stratégies gagnantes, mais c'est aussi lui qui reconnaît ou non comme telles ces stratégies. » (p. 11).

\section{Jeu d'apprentissage}

La notion de jeu d'apprentissage permet de rendre compte de la succession de "scènes » dans une séquence d'enseignement/apprentissage. Ces scènes 
sont définies comme étant « connexes et clos sur elles-mêmes » (Sensevy, 2007; p. 16) et se « délimitent en général par une «entrée en matière »-qui annonce la nouvelle scène et la démarque de la précédente, et par une « conclusion»» (Sensevy, 2007; p. 16).

Ces jeux d'apprentissage se distinguent les uns des autres par le contrat didactique en jeu ainsi que par le milieu. Une façon de décrire ces jeux, afin de pouvoir les comparer et de les distinguer les uns des autres, est d'utiliser le triplet des genèses: la chronogenèse, la mésogenèse et la topogenèse.

\section{Chronogenèse}

Pour répondre à la question " comment quand? » il faut chercher à mettre en évidence comment le savoir évolue avec le temps dans la classe. L'enseignement est vu comme une évolution, les savoirs étant disposés le long d'un axe temporel (temps des horloges) ils sont constitutifs d'un temps-savoir (temps didactique). D'une classe à l'autre, le rythme d'introduction des savoirs varie (Andrée Tiberghien, Malkoun, Buty, Souassy, \& Mortimer, 2007). Lavancement du savoir dans la classe est dû autant aux élèves (contraints par leurs temps d'apprentissage) qu'à l'enseignant (gestionnaire du temps d'enseignement). Imaginons une classe où les élèves ont des difficultés à comprendre les notions en jeu, ils peuvent adopter diverses attitudes dont les deux ci-dessous qui nous paraissent majeures:

- les élèves peuvent interrompre l'enseignant pour demander des explications ou engager d'autres formes de transaction moins explicites mais qui toutes tendent à ralentir le rythme d'introduction de nouveaux savoirs;

- les élèves peuvent aussi se positionner en retrait, et attendre de voir s'ils vont comprendre « à tête reposée » chez eux; l'enseignant continue alors à avancer dans le savoir sans être interrompu et la plupart du temps sans se douter de la portée des difficultés rencontrées par les élèves ou par certains d'entre eux.

\section{Topogenèse}

La topogenèse permet de rendre compte de la distribution des rôles entre les acteurs de la classe. Cette répartition est le fruit d'une négociation qui précise les tâches dévolues à l'élève et celles dévolues à l'enseignant. L'ensemble de ces tâches définit la position (le topos) de chacun par rapport au savoir. Sensevy (2007) donne l'exemple d'une dictée au cours de laquelle l'enseignant décide d'écrire au tableau un mot de la dictée qu'il juge difficile. En agissant ainsi l'enseignant redéfinit la tâche de l'élève, celui-ci n'a plus à savoir écrire correctement le mot, mais à savoir le recopier.

\section{Mésogenèse}

La mésogénèse s'intéresse au renouvellement du milieu. Dans les travaux de Piaget le milieu est vu comme un contexte cognitif de l'action (Sensevy, 2007). Il s'agit d'un contexte cognitif commun ainsi que des objets matériels grâce auxquels l'action d'enseigner et d'apprendre peut avoir lieu. Si l'on reprend l'exemple de la dictée, lorsque l'enseignant annonce aux élèves qu'ils vont faire une dictée, un contexte cognitif commun est instancié. Ainsi les élèves et l'enseignant se retrouvent placés dans un contexte comportant ses règles, codes... Pour Brousseau (1998) le milieu devrait être, autant que possible, antagoniste, c'est-à-dire que l'élève, pour résoudre un problème (au sens large) agit sur le milieu et reçoit des rétroactions qui lui permettent de trouver la solution au problème.

La mésogenèse est la catégorie permettant de répondre à la question « Comment quoi?». C'està-dire qu'il s'agit de rendre compte de l'évolution du milieu, comment l'enseignant va introduire dans le milieu une signification, avec quel énoncé, avec quel(s) objet(s).

Cet ensemble de descripteurs permet de rendre compte de l'action professorale dans la classe, ce que Sensevy (2007) considère comme l'action visant à «faire jouer le jeu ». Laction didactique prend également en compte l'intentionnalité du professeur. Ainsi pour Schubauer-Leoni et al (2007) l'action conjointe est guidée par une intention conjointe, qui est caractérisée par une interdépendance de buts, distincts pour l'élève et pour l'enseignant. Deux autres points de vue sur l'action du professeur sont pris en compte en considérant, premièrement, la «préparation du jeu » et, deuxièmement, les « déterminants du jeu ». 


\section{Caractérisation du savoir}

Le savoir en jeu est fondamental dans la TACD, il semble donc important de caractériser ce savoir.

La modélisation est un aspect fondamental du fonctionnement des sciences. Le rapport entre le concret et l'abstrait et l'articulation entre expérimental et théorique est en effet au cœur de ces disciplines. La recherche en didactique s'est penchée sur cet aspect du fonctionnement des sciences (Martinand, 1992; Tiberghien, 1994, Sensevy et al. 2008), afin de proposer des séquences d'enseignement ou d'analyser des situations (Tiberghien,
Vince, \&, Gaidioz, 2009). Ces travaux proposent un modèle du fonctionnement de la physique enseignée en considérant deux mondes. Le monde des objets et événements qui correspond aux aspects observables du monde matériel auquel fait écho le monde des théories et modèles dans lequel se trouvent les principes, les paramètres et les quantités. Une adaptation de ce modèle à la chimie (Le Maréchal, 1999), permet de prendre en compte une particularité de cette discipline: les chimistes ont souvent recours à une explication microscopique du monde matériel et utilisent, de ce fait, des notions qui ont un statut d'objets dans un monde qui est non-perceptible (Le Maréchal \& Bécu-Robinault, 2006).
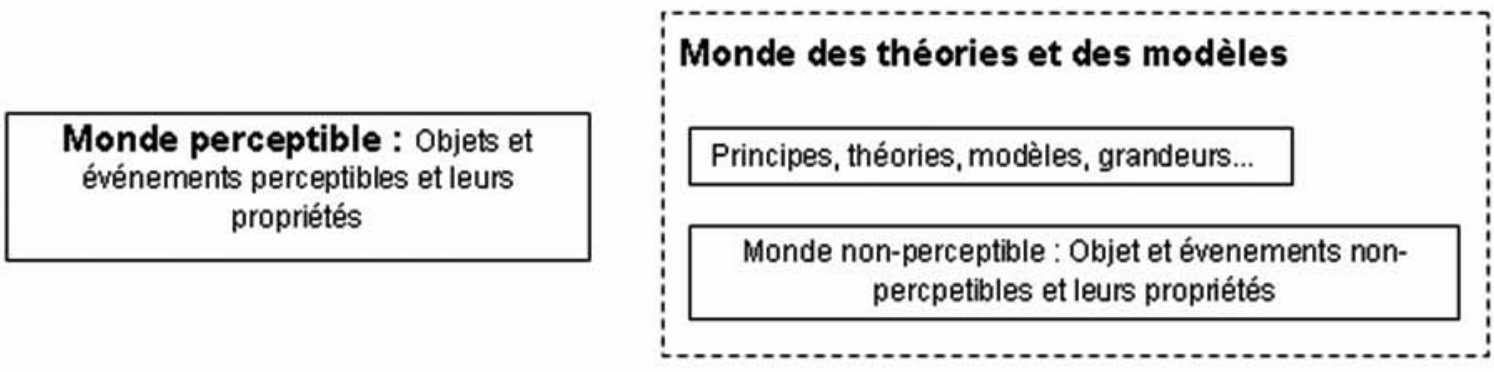

Figure 2: Représentation des différents mondes (J.-F. Le Maréchal \& Bécu-Robinault, 2006).

Ainsi, le changement de couleur d'une solution est un événement du monde perceptif interprétable dans le monde non-perceptible en termes d'ions, de molécules et de chocs entre particules: une solution bleue qui devient incolore peut être interprétée comme la disparition des ions cuivre contenus dans cette solution.

\section{Articulation entre TACD et PCK}

Lépistémologie pratique du professeur est un déterminant de l'action didactique qui fait résonance aux connaissances professionnelles. Cette épistémologie est pratique car elle intervient dans le vif des actions du professeur sans que ce dernier en soit nécessairement conscient. Cette épistémologie est donc construite dans et à partir de la pratique d'enseignement. En plus d'une théorie sur ce qu'est la chimie (pour notre cas), l'enseignant développe, à partir de son action, une théorie-pratique de ce qu'est enseigner la chimie. Dans le cadre de la TACD, les connaissances professionnelles sont ainsi abordées comme des déterminants de l'action didactique.

Notre but est de décrire l'action de l'enseignant dans sa classe, autrement dit de prendre le point de vue de l'enseignant lorsqu'il joue le jeu dans la classe, en fonction des connaissances professionnelles mises en œuvre. Les connaissances ainsi reconstruites émergent de l'action didactique. Il s'agit ainsi, comme dit plus haut d'étudier des connaissances dans l'action et non plus des connaissances sur l'action. Le statut des connaissances étudiées se trouve ainsi changé. La question qui se pose alors est de savoir si le système de catégories mis au point par l'approche PCK reste pertinent pour étudier les connaissances reconstruites dans le cadre de la TACD. Ce que nous nous proposons de faire, en d'autres mots, est d'importer la liste de ces connaissances et de leurs caté- 
gories mises au point par l'approche en termes de PCK dans le cadre de la TACD. Cette catégorisation des connaissances sera ainsi discutée du point de vue de la TACD.

\section{Méthodologie}

Nous avons mené une étude de cas correspondant à l'observation d'une enseignante de chimie en classe de Terminale S. Nos données sont constituées principalement d'enregistrements vidéo pris en classe ordinaire. Ces données vidéo ont un volume horaire d'environ 25 heures et correspondent à l'observation de 10 séances en classe entière et de 8 séances de travaux pratiques en demi-classe. L'enseignement porte sur la notion de constante d'équilibre dans le cas des acides et des bases.

Notre méthodologie s'appuie sur une double approche: une analyse narrative d'extraits de bandes vidéo et une analyse statistique de mots clés attribués à ces mêmes extraits vidéo. Cette double approche n'est pas envisagée comme une triangulation des analyses (où l'analyse statistique viendrais confirmer l'analyse narrative par exemple), mais plutôt comme un aller retour entre ces deux types d'analyse afin qu'elles se nourrissent l'une l'autre. Ce dispositif permet de rendre compte de l'aspect générique ou spécifique de l'analyse narrative grâce à l'analyse statistique, et de donner du sens à l'analyse statistique grâce à l'analyse narrative. Cette analyse est menée sur les données vidéo prises en classe entière (les séances de travaux pratiques n'ont pas été analysées).

\section{Structuration du corpus}

Après avoir recueilli les données vidéos, les productions verbales des acteurs de la classe ont été transcrites (il s'agit d'une transcription relativement grossière). Puis le corpus a été structuré grâce à un découpage en thèmes. Le thème est une unité du discours lors duquel celui-ci est centré sur un objet de savoir. Chaque thème a une durée variable pouvant aller de quelques minutes à plusieurs dizaines de minutes,. Le repérage des thèmes, c'est-à-dire la détermination de leurs frontières, est facilité par la présence de marqueurs linguistiques. Par exemple lorsque l'enseignante dit: «alors là je pense qu'on a répondu du coup aux trois premiers exercices [...] donc on passe à la deuxième partie c'est le vif du sujet », l'attention du chercheur se porte sur ce passage en particulier. Toutefois, les marqueurs langagiers seuls ne permettent pas de conduire le découpage en thèmes, le savoir enseigné en jeu reste l'élément principal pour déterminer lorsqu'il y a changement de thème. Lintérêt de ce découpage en thèmes est qu'il permet une lecture du corpus du point de vue du savoir enseigné. L'attention est ainsi portée sur l'enjeu de savoir, sur ce qui se passe dans la classe du point de vue didactique. De plus le thème est une unité suffisamment grande pour avoir une vision d'ensemble du corpus (environ 25 heures de vidéo), tout en permettant de voir, au sein d'une séance (en moyenne d'une durée d'une heure) l'évolution du savoir via les thèmes.

\section{Sélection de moments propices à la reconstruction de Teacher Pedagogical Constructions}

Après avoir structuré le corpus, nous avons sélectionné des moments propices à la reconstruction de TPC. Cette étape repose sur l'hypothèse suivante: certains moments de la classe sont des moments privilégiés pour la mobilisation, par l'enseignante, de PCK et donc de Teacher Pedagogical Constructions (TPC). En partant de la remarque de van Driel et al. (1998) selon laquelle les deux principales catégories de TPC sont les connaissances relatives aux difficultés d'élèves et les connaissances relatives aux stratégies d'enseignement, les moments qui correspondent à une intervention d'un élève (question, remarque ou constatation par exemple) et où l'enseignante y répond sont supposés être des moments propices à la mise en ouvre de TPC. En effet il est raisonnable de penser que pour répondre à une intervention d'élève l'enseignante cherche à cibler la difficulté à l'origine de cette intervention, pour cela elle met en œuvre une TPC « difficulté d'élève ». De plus lorsque l'enseignante répond à une intervention d'élève, il est probable qu'elle mette en œuvre une stratégie, et donc une TPC «Stratégie d'enseignement». Ces passages correspondant à une intervention d'élève ainsi que la réponse de l'enseignante nous les avons appelés « épisodes interactionnels, ».

Les épisodes interactionnels présentent l'avantage d'être des moments de la classe ayant un début 
et une fin clairement marqués (le cours « normal » de la classe est interrompu par une intervention d'élève. Une fois l'épisode fini, le cours « normal » reprend). Ces épisodes présentent également une certaine homogénéité: ils sont tous initiés par un élève, et leur fonctionnement est régi par un même aspect du contrat didactique de la classe. En effet ces épisodes apparaissent dans la classe car les élèves ont le droit d'interrompre l'enseignante lorsqu'ils ont une question à poser ou une remarque à faire, l'enseignante, quand à elle, répond à toute les interventions d'élèves.

Ces épisodes ne sont pas autonomes, ils s'intègrent dans une histoire de la classe, dans un certain contexte. Le découpage en thèmes permet de rendre compte de cette histoire du savoir dans la classe, et ainsi d'interpréter ce qui se passe dans les épisodes interactionnels.

\section{Intentionnalité de l'enseignante}

Lors de la prise de données, nous avons été frappé par le fait que l'enseignante proposait aux élèves un devoir de type « $\mathrm{Bac}$ » et un devoir de type non-Bac (ce sont les termes de l'enseignante). En effet cette enseignante enseigne la chimie en classe de Terminale. Du point de vue de la TACD, l'existence de ces deux types de sujets peut être interprétée en termes d'intentionnalité de l'enseignante qui cherche à atteindre deux buts: préparer les élèves au Bac et faire en sorte que les élèves apprennent de la chimie.

\section{Mots clés}

Notre méthodologie a été construite à l'aide du logiciel Transana, qui est un logiciel d'analyse qualitative des vidéos. Ce logiciel offre la possibilité d'affecter des mots clés à des extraits vidéo, nous avons saisi cette opportunité pour affecter des mots clés à nos épisodes interactionnels. Ces mots clés sont issus directement de notre cadre théorique et prenne en compte la spécificité de l'action didactique comme définie dans la théorie de l'action conjointe. Ainsi ces mots clés vont permettent de rendre compte:

- Des buts à l'œuvre dans l'épisode interactionnel (Préparer les élèves au Bac? Faire en sorte que les élèves apprennent de la chimie? Les deux buts à la fois?).

- Du type de savoir en jeu dans l'épisode (A quel niveau de modélisation appartient le savoir en jeu dans la question de l'élève et dans la réponse de l'enseignante)

- De la façon dont le savoir est mis en œuvre:

- Du point de vue de la chronogenèse, il s'agit de regarder si l'enseignante introduit un savoir nouveau pour répondre à la question de l'élève, si elle fait un rappel. L'enjeu est notamment de suivre l'« histoire » des objets matériels (inscriptions au tableau, manuels scolaires) utilisés pour répondre à l'élève... Cette dynamique d'apparition des objets matériels? sera traitée dans cette étude du point de vue? de la chronogenèse dans le sens où elle permet de rendre compte de l'apparition et de la disparition d'élément de savoir en fonction du temps, mais n'est pas interprétée en termes de la façon dont les objets contribuent à construire du sens pour les élèves (le « comment quoi » de la mésogenèse).

- Du point de vue de la topogenèse, il s'agit de prendre en compte l'implication, au travers de l'analyse du discours des épisodes interactionnels, des acteurs de la classe dans la construction et l'évolution du savoir.

Lanalyse en termes de mésogenèse, contrairement à celles en termes de topo et chronogenèse, se prête plus difficilement à une catégorisation en mots clés. Pour pouvoir être pertinente la catégorisation en termes de mots clés ne doit pas comporter un trop grand nombre de mots clés différents, sinon il devient difficile de voir " apparaitre » des patterns ou des configurations de mots clés. Alors que des méta catégories ont pu être construites pour l'analyse en termes de chrono et topogenèse (voir infra), qui permettent de rendre compte avec un nombre relativement restreint de mots clés des situations observées, ce passage d'une dimension de description à des méta catégories est plus délicat dans le cas de la mésogenèse. En effet comment rendre compte de la diversité des situations $\mathrm{du}$ « comment quoi » qui peuvent se produire avec un nombre limité de mots clés? Nous avons donc choisi de ne pas prendre en compte cette dimension de l'ana- 
lyse à l'état actuel de notre recherche, tout en gardant à l'esprit que les trois dimensions de description du jeu didactique (chrono, topo et mésogenèse) sont liées entre elles. Cette limitation de notre méthodologie n'est donc pas sans conséquence sur la compréhension de l'action professorale.

Nous détaillons l'analyse qui permet d'attribuer aux épisodes interactionnels les mots clés de ces deux dernières catégories (chronogenèse et topogenèse).

\section{Catégorisation en termes de chronogenèse}

Notre catégorisation chronogénétique de la vie du savoir contient deux aspects. Un premier aspect est relatif à la gestion, par l'enseignante, de l'avancée du savoir dans la classe. Nous cherchons à décrire comment l'enseignante « intègre » le savoir en jeu dans l'épisode interactionnel par rapport à l'avancée du savoir. En effet l'enseignante ne peut prévoir quel savoir va être en jeu dans l'épisode interactionnel, puisque c'est l'élève qui pose la question. Il lui appartient donc de gérer ce savoir du point de vue de sa planification. Ainsi l'enseignante dispose de plusieurs choix: elle peut décider de ne pas répondre tout de suite à la question de l'élève et d'attendre un moment plus propice (par exemple lorsqu'elle aura introduit, entre-temps, de nouveaux objets de savoir). Elle peut également décider de répondre tout de suite à la question de l'élève. Pour rendre compte de cet aspect de l'action de l'enseignante, il nous faut prendre en compte le savoir en jeu dans la question de l'élève par rapport au savoir en jeu dans le thème dans lequel s'inscrit l'épisode interactionnel. En effet nous faisons l'hypothèse que l'enseignante ne va pas gérer de la même façon l'avancée du savoir selon que le savoir en jeu dans l'épisode appartient au thème ou au contraire n'y appartient pas. Nous différencions donc d'une part les épisodes interactionnels dont le savoir appartient au thème dans lequel l'épisode apparaît, et d'autre part les épisodes dont le savoir en jeu n'appartient pas au thème. Les exemples suivants illustrent les différents cas de figures mentionnés ci-dessus:

\section{Exemple 1:}

Cet épisode interactionnel se situe dans le troisième thème de la séance 03 . Le thème a pour titre: Acide base et réactions acido-basiques.
Locuteur Productions verbales
$\mathrm{P}$ acide chlorhydrique oui
E j'ai pas bien compris le couple $\mathrm{H}_{3} \mathrm{O}+\mathrm{H}_{2} \mathrm{O}$ / parce qu'en fait c'est $\mathrm{H}_{2} \mathrm{O}$ égale heu non c'est $\mathrm{H}_{2} \mathrm{O}$ plus $\mathrm{H}^{+}$égale $\mathrm{H}_{3} \mathrm{O}^{+}$

$\mathrm{P} \quad$ oui on va l'écrire juste maintenant

E mais c'est vrai qu'il y a un $\mathrm{H}^{+}$déjà de (...?) parce qu' $\mathrm{H}_{3} \mathrm{O}+$ c'est pas (...?) /

$\mathrm{P}$ donc ici on va voir justement c'est les problèmes d'écriture de l'ion oxonium/alors on va écrire déjà ça et je réponds à ta question juste après donc dans le cas de l'acide chlorhydrique ça veut dire ce qu'on a fait en TP la première mesure quel est le premier couple mis en jeu qu'est ce qu'on a dissous

\section{Extrait 1: Transcription d'un épisode interactionnel ou l'enseignante reporte sa réponse à plus tard.}

Dans cet exemple l'enseignante répond à l'élève qu'ils traiteront de cette question par la suite et qu'elle veut d'abord finir ce qu'ils sont en train de faire. Le savoir en jeu dans la question (l'ion oxonium) fait partie du thème (Acide, base et réaction acido-basique). Vraisemblablement l'enseignante avait prévu de traiter cette question. Elle décide de reporter la réponse à la question de l'élève, l'enseignante répond dans ce même thème.

\section{Exemple 2:}

$$
\begin{aligned}
& \text { Locuteur Productions verbales } \\
& \mathrm{E} \quad \mathrm{y} \text { a le } \mathrm{pH} \text { qui correspond au point d'équivalence c'est } \\
& \text { le } \mathrm{pK}_{\mathrm{a}} \text { non } \\
& \mathrm{P} \quad \text { non pas force heu non je crois pas forcément }
\end{aligned}
$$

Extrait 2: transcription d'un épisode interactionnel ou l'enseignante ne développe pas sa réponse.

Dans cet exemple l'enseignante donne une réponse sans la développer (elle aurait pu donner un exemple). Le savoir en jeu dans l'épisode interactionnel fait partie du thème.

Le deuxième aspect de la chronogénèse que nous prenons en compte est la position du savoir en jeu dans la réponse de l'enseignante par rapport à l'histoire du savoir dans la classe. En effet le savoir qui vit dans la classe s'inscrit dans le temps. Du point de vue des élèves, certains savoirs sont nouveaux, d'autres sont plus anciens ayant déjà été introduits 
dans la classe. Enfin d'autres savoirs peuvent faire le lien entre des savoirs anciens et nouveaux. Nous distinguons les savoirs anciens qui ont été introduits dans des classes précédentes (en classe de seconde ou de première par exemple) des savoirs anciens introduits dans un thème précédent. L'épisode dont la transcription est donnée dans l'extrait 3 montre un exemple où l'enseignante fait un lien entre un savoir nouveau et un savoir ancien.

$$
\begin{aligned}
& \text { Locuteur Productions verbales } \\
& \text { E madame } \\
& \text { P oui } \\
& \text { E ça veut dire c'est pas possible d'avoir une }
\end{aligned}
$$
solution neutre avec une concentration de 10 moins 3 et 10 moins 3 pour heu

$\mathrm{P}$ pas à 25 degré/d'accord à 25 degré on va avoir si y a une solution neutre $\mathrm{HO}^{-}$égale $\mathrm{H}_{3} \mathrm{O}^{+}$et donc $\mathrm{K}_{\mathrm{e}}$ ' qui vaut $\mathrm{H}_{3} \mathrm{O}^{+}$fois $\mathrm{HO}^{-}$c'est 10 moins 14 donc si on a 10 moins 3 et 10 moins 3 on est pas à l'équilibre

Extrait 3: Transcription d'un épisode interactionnel lors duquel l'enseignante fait le lien entre un savoir ancien et un savoir nouveau.

Dans une solution contenant des ions $\mathrm{H}_{3} \mathrm{O}^{+}$et $\mathrm{HO}^{-}$le produit de la concentration en ion $\mathrm{H}_{3} \mathrm{O}^{+}$par la concentration en ion $\mathrm{HO}^{-}$est égale à une constante notée $\mathrm{K}_{\mathrm{e}}$. Cette constante ne dépend que de la température. Ainsi à 25 degrés cette constante a une valeur de $10^{-14}$. Dans une solution neutre la concentration en ion $\mathrm{H}_{3} \mathrm{O}^{+}$est égale à la concentration en ion $\mathrm{OH}^{-}$.

Les élèves ont vu que la constante $\mathrm{K}_{\mathrm{e}}$ ne dépend que de la température et que sa valeur à 25 degrés est de $10^{-14}$.

L'élève veut savoir s'il est possible de disposer d'une solution neutre dont les concentrations en ion $\mathrm{H}_{3} \mathrm{O}^{+}$et $\mathrm{OH}^{-}$sont égales à $10^{-3} \mathrm{~mol}$ par litre. L'enseignante rappelle qu'à 25 degré $\mathrm{K}_{\mathrm{e}}$, qui est égale au produit des concentrations en ion $\mathrm{H}_{3} \mathrm{O}$ + et $\mathrm{OH}^{-}$, est égale à $10^{-14}$ (savoir ancien). Elle finit sa réponse en disant que si les concentrations sont égales à $10^{-3}$ le système n'est pas à l'équilibre (savoir nouveau).

Mots clés: Nous avons construit une catégorie de mots clés suivant les deux aspects retenus, l'intégration du savoir en jeu dans l'épisode par rapport au savoir en jeu dans le thème et le statut du savoir en jeu par rapport à l'histoire du savoir dans la classe. Cette catégorie est centrée sur l'action de l'enseignante. Une catégorie de mots clés a également été créée pour tenir compte de la «place » du savoir dans la question de l'élève par rapport au découpage thématique. Ces catégories ainsi que les mots clés sont donnés dans le tableau 1.

\begin{tabular}{|l|l|}
\hline \multicolumn{1}{|c|}{ Catégorie } & \multicolumn{1}{c|}{ Mot clé } \\
\hline \multirow{4}{*}{$\begin{array}{l}\text { Chronogénèse } \\
\text { (réponse de } \\
\text { l'enseignante) }\end{array}$} & $\begin{array}{l}\text { Lien savoir nouveau-ancien (quel que soit } \\
\text { le savoir ancien) }\end{array}$ \\
\cline { 2 - 2 } & Se développe pas la réponse \\
\cline { 2 - 2 } & Savoir nouveau sans lien ancien, autre classe sans lien \\
\cline { 2 - 2 } & Savoir ancien, autre thème sans lien \\
\hline \multirow{2}{*}{$\begin{array}{l}\text { Chronogénèse } \\
\text { (question de de }\end{array}$} & Appartient au thème \\
\cline { 2 - 2 } l'élève) & N'appartient pas au thème \\
\hline
\end{tabular}

Tableau 1 : catégories de mots clés et mots clés construits à partir de l'analyse en termes de chronogénèse.

\section{Catégorisation en termes de topogénèse}

La topogénèse peut être définie comme la position des acteurs de la classe par rapport au savoir, c'est-àdire à qui incombe la responsabilité de faire évoluer le savoir dans la classe. Nous avons choisi de rendre compte de la topogénèse en analysant les interactions entre l'élève et l'enseignante dans les épisodes interactionnels. Pour cela nous avons utilisé le modèle de Sinclair et Coulthard (1975) qui a été conçu pour analyser les interactions orales, en particulier dans les situations de classe.

\section{Modèle de Sinclair et Coulthard}

Ce modèle définit plusieurs niveaux d'analyse, mais nous n'en avons retenu que trois: l'acte, le mouvement et l'échange. Un échange est composé de mouvements, et un mouvement est composé d'actes. Nous ne détaillerons pas l'ensemble du modèle, mais seulement les points qui se sont révélés pertinents pour notre étude. 
Pour Sinclair et Coulthard les échanges en classe peuvent être modélisés par une structure ternaire notée IRF (Initiation, Response, Feedback). Linitiation, la réponse et le feedback sont des mouvements qui peuvent être caractérisés par leur acte principal. Le tableau 2 présente les actes que nous avons retenus pour nos analyses parmi les vingt deux identifiés par Sinclair et Coulthard:

\begin{tabular}{|c|c|l|}
\hline Mouvement & Acte & \multicolumn{1}{|c|}{ Description } \\
\hline \multirow{3}{*}{ Initiation } & Incitation & Mouvement qui demande une réponse \\
\cline { 2 - 3 } & Informer & Mouvement qui fait part d'une information \\
\hline \multirow{2}{*}{ Réponse } & Informer & Mouvement qui fait part d'une information \\
\cline { 2 - 3 } & Accepter & Mouvement qui valide une information \\
\cline { 2 - 3 } & Incitation & La réponse se fait sous forme de question \\
\hline \multirow{2}{*}{ Feedback } & Acknowledge & Signe que la réponse a été entendue \\
\cline { 2 - 3 } & Accepter & La réponse est acceptée \\
\hline
\end{tabular}

Tableau 2: Mouvements et actes de la classification de Sinclair et Coulthard retenus.

Les échanges sont constitués d'un enchaînement de mouvements. Sinclair et Coulthard décrivent plusieurs types d'échanges, mais là encore nous présentons seulement ceux qui ont été utilisés dans le cadre de ce travail.

- Léchange de sollicitation a pour but d'obtenir une réponse, il est très souvent caractérisé par une structure IRF si c'est l'enseignant qui pose la question, et par une structure IR si c'est l'élève qui pose la question.

- Léchange d'information a pour but de valider une information. Il a la plupart du temps une structure IR.

Le tableau 3 montre les catégories que nous avons identifiées lors de notre analyse:

\begin{tabular}{|l|l|}
\hline \multicolumn{1}{|c|}{ Échange } & \multicolumn{1}{c|}{ Description } \\
\hline Sollicitation élève & L'élève pose une question, l'enseignante répond. \\
\hline Sollicitation enseignant & L'enseignante pose une question, les élèves répondent, l'enseignante valide la réponse. \\
\hline Information élève & L'élève donne une information, l'enseignante la valide. \\
\hline Information enseignante & L'enseignante donne une information, les élèves peuvent donner une rétroaction \\
\hline
\end{tabular}

Tableau 3: Catégories d'échanges

\section{Caractérisation des épisodes interactionnels}

Un épisode interactionnel est, la plupart du temps, composé de plusieurs échanges. Nous avons caractérisé ces épisodes en fonction du type d'échange:
Une seule sollicitation d'élève

Un exemple est donné dans le tableau 4 qui indique les différents types d'Échanges, de Mouvements et d'Actes.

\begin{tabular}{|l|l|l|l|l|}
\hline Locuteur & Production verbale & Échange & Mouvement & Acte \\
\hline E & il sert à quoi en fait heu K & & Initiation & Incitation \\
\cline { 1 - 5 } $\mathrm{P}$ & $\begin{array}{l}\text { alors à quoi sert K/alors Taux on a compris à quoi ça servait K } \\
\text { bah en fait donc K on a dit c'est indépendant/d'état initial ça veut } \\
\text { dire si on a la transformation on connaît la valeur de K/et K ça } \\
\text { relie les concentrations à l'état final/donc ça va nous permettre } \\
\text { aussi de revenir à taux }\end{array}$ & Sollicitation élève & Réponse & Information \\
\hline
\end{tabular}

Tableau 4: Exemple d'un épisode interactionnel composé d'un échange de sollicitation d'élève. 
Plusieurs sollicitations d'élèves.

\begin{tabular}{|c|c|c|c|c|}
\hline Locuteur & Production verbale & Échange & Mouvement & Acte \\
\hline E & mais ça représente quoi en fait $K$ & \multirow[t]{2}{*}{ Sollicitation élève } & Initiation & Incitation \\
\hline $\mathrm{P}$ & bah ça représente l'équilibre & & Réponse & Information \\
\hline E & c'est pareil pour n'importe quel acide & \multirow[b]{2}{*}{ Sollicitation élève } & Initiation & Incitation \\
\hline $\mathrm{P}$ & $\begin{array}{l}\text { non c'est pas pareil enfin chaque acide va avoir sa constante } \\
\text { d'équilibre d'accord donc si vous regardez justement dans votre } \\
\text { livre dans le rabat de couverture le premier vous avez/les couples } \\
\text { acide base en solution aqueuse/on vous donne leurs noms et leurs } \\
\text { formules/et on vous donne } \mathrm{K}_{\mathrm{a}} / \mathrm{d} \text { 'accord c'est } \mathrm{K}\end{array}$ & & Réponse & Information \\
\hline
\end{tabular}

Tableau 5: Épisode interactionnel constitué de plusieurs échanges de sollicitations successives d'élèves

\section{Sollicitations d'élèves et information de l'enseignante}

Lépisode interactionnel est composé d'une ou plusieurs sollicitations d'élèves les unes à la suite des autres suivies d'une information de l'enseignante.
Un exemple est donné dans le tableau 6. Nous n'analysons pas le début de l'épisode où l'enseignante demande à l'élève de répéter sa question.

\begin{tabular}{|c|c|c|c|c|}
\hline Locuteur & Production verbale & Échange & Mouvement & Acte \\
\hline E & $\begin{array}{l}\text { est ce que }[\ldots] \text { vu que }[\ldots] \text { comme on a } \mathrm{V} \text { toujours un litre on va } \\
(\ldots \text { ?) toujours pareil }(\ldots ?)\end{array}$ & & & \\
\hline $\mathrm{P}$ & de quoi & & & \\
\hline E & $\begin{array}{l}\text { comme } \mathrm{V} \text { c'est un litre même si on divise à chaque fois par le } \\
\text { volume on va trouver }\end{array}$ & \multirow{3}{*}{ Sollicitation élève } & Initiation & Incitation \\
\hline $\mathrm{P}$ & ah bah oui mais la valeur oui c'est la même & & Réponse & Informer \\
\hline E & oui la valeur oui c'est la même & & Feedback & Aknowledge \\
\hline $\mathrm{P}$ & $\begin{array}{l}\text { mais si on avait pris } 500 \text { millilitres du coup on aurait eu des } \\
\text { valeurs qui changent mais comme on cherche des concentrations } \\
\text { autant prendre un litre comme ça c'est plus simple }\end{array}$ & \multirow[t]{2}{*}{$\begin{array}{l}\text { In for m a tio } \mathrm{n} \\
\text { enseignante }\end{array}$} & Initiation & Informer \\
\hline E & ouais// & & Feedback & Accepter \\
\hline
\end{tabular}

Tableau 6: Épisode interactionnel composé d'une sollicitation d'élève suivie d'une information de l'enseignante. 


\section{Sollicitation d'un élève et sollicitation de l'enseignante}

Lépisode est composé d'une ou plusieurs sollicitations d'élèves les unes à la suite des autres suivies d'une sollicitation de l'enseignante.

Voici un exemple:

\begin{tabular}{|c|c|c|c|c|}
\hline Locuteur & Production verbale & Échange & Mouvement & Acte \\
\hline E & est-ce que ça pourrait être possible aussi une courbe dans l'autre sens & \multirow{2}{*}{ Sollicitation élève } & Initiation & Incitation \\
\hline $\mathrm{P}$ & oui & & Réponse & Informer \\
\hline $\mathrm{P}$ & ça veut dire quoi si on a une courbe dans l'autre sens// & \multirow[b]{3}{*}{$\begin{array}{l}\text { Sollicitation } \\
\text { enseignante }\end{array}$} & Initiation & Incitation \\
\hline E & $(\ldots ?)$ & & Réponse & Informer \\
\hline $\mathrm{P}$ & $\begin{array}{l}\text { on pourrait avoir ça là ça veut dire on a une solution au départ qui est } \\
\text { basique ça veut dire on dose en fait une solution basique et on ajoute } \\
\text { de l'acide d'accord donc après quand le réactif limitant c'est la base } \\
\text { on a une solution acide d'accord donc on peut avoir ça va nous dire } \\
\text { la forme de la courbe qu'elle espèce chimique on dose ça veut dire } \\
\text { qu'est ce qui est présent au départ dans le Bêcher/ }\end{array}$ & & Feedback & Informer \\
\hline
\end{tabular}

Tableau 7 : Épisode interactionnel composé d'une sollicitation d'élève suivie d'une sollicitation de l'enseignante.

\section{Sollicitation d'un élève et sollicitation de l'enseignante enchâssée}

Lépisode est composé d'une sollicitation d'élève dans laquelle est enchâssée une sollicitation de l'enseignante. Un exemple permet de mieux comprendre:

\begin{tabular}{|c|c|c|c|c|c|}
\hline Locuteur & Production verbale & \multicolumn{2}{|l|}{ Échange } & Mouvement & Acte \\
\hline E & $\begin{array}{l}\text { madame quand on a un } \mathrm{pK}_{\mathrm{a}} \text { on dit heu à } \\
7,5 \text { on place à } \mathrm{pH} 7 \text { égale à } 7,5\end{array}$ & \multirow{2}{*}{\multicolumn{2}{|c|}{ Sollicitation élève }} & Initiation & Incitation \\
\hline $\mathrm{P}$ & $\begin{array}{l}\text { à pH égale à } 7,5 \text { ça sera la séparation des } \\
\text { domaines de prédominances }\end{array}$ & & & Réponse & Informer \\
\hline $\mathrm{E}$ & $\begin{array}{l}\text { pourquoi avec l'eau pourquoi le } \mathrm{pK}_{\mathrm{a}} \text { est } \\
\text { égal à } 14 \text { si c'est neutre à } 7 \text { à } \mathrm{pH} \text { égal } 7\end{array}$ & \multirow{4}{*}{ Sollicitation élève } & & Initiation & Incitation \\
\hline $\mathrm{P}$ & $\begin{array}{l}\text { parce qu'avec l'eau on n'a pas écrit } \mathrm{K}_{\mathrm{a}} \text { on } \\
\text { a écrit quoi }\end{array}$ & & \multirow{3}{*}{$\begin{array}{l}\text { Sollicitation } \\
\text { enseignante }\end{array}$} & Réponse & Incitation \\
\hline E & $\mathrm{pK}_{\mathrm{e}}$ & & & Réponse & Informer \\
\hline $\mathrm{P}$ & $\mathrm{K}_{\mathrm{e}} /$ & & & Feedback & Accepter \\
\hline
\end{tabular}

Tableau 8: Épisode interactionnel composé d'une sollicitation de l'enseignante enchâssée dans une sollicitation de l'élève. 
Nous avons ainsi caractérisé les épisodes interactionnels, du point de vue de l'analyse de la conversation, en cinq types: une seule sollicitation d'élève, plusieurs sollicitations d'un élève, sollicitation d'un élève suivie de sollicitation de l'enseignante et sollicitation de l'enseignante enchâssée dans une sollicitation de l'élève. Nous faisons l'hypothèse que cette analyse en termes de conversation permet de rendre compte de la topogénèse des épisodes interactionnels. Si, par définition de l'épisode interactionnel, l'élève est toujours l'initiateur, l'action de l'enseignante quant à elle peut être variée. Lorsque l'épisode est de type une seule ou plusieurs sollicitations d'élève, l'enseignante prend en charge de donner la réponse; les élèves sont en charge de l'objet de la question et désignent dès lors l'élément de savoir en jeu dans l'épisode interactionnel. Lorsque l'épisode interactionnel est de type sollicitation d'élève suivie de sollicitation de l'enseignante ou sollicitation de l'enseignante enchâssée dans une sollicitation d'élève, l'enseignante renverse la situation: les élèves sont en charge de fournir une réponse, et l'enseignante oriente les élèves vers la réponse. Le cas des épisodes de type sollicitation d'élève suivie d'une information de l'enseignante montre un autre partage des rôles: l'enseignante est en charge de la réponse mais ne veut pas se contenter de la seule réponse à la question de l'élève, elle ajoute une information, reliée à l'élément de savoir en jeu dans l'épisode interactionnel, et qui complète la réponse.

Mots clés:

Cette analyse nous a permis de créer une catégorie de mots clés liée à la topogenèse. Les mots clés de cette catégorie sont présentés dans le tableau ci dessous:

\begin{tabular}{|l|l|}
\hline Catégorie & Mot clé \\
\hline \multirow{4}{*}{ Topogénèse } & Une seule sollicitation d'élève \\
\cline { 2 - 3 } & Plusieurs sollicitations d'élève \\
\cline { 2 - 3 } & Sollicitations d'élève suivie de sollicitation de l'enseignante \\
\cline { 2 - 3 } & Sollicitation d'élèves suivie d'information de l'enseignante \\
\cline { 2 - 3 } & Sollicitation de l'enseignante enchâssée dans sollicitation élève \\
\hline
\end{tabular}

Tableau 9: Mots clé de la catégorie topogenèse.

Nous obtenons, au terme de ces différentes étapes, une base de données d'extraits de vidéo indexés suivant des mots clé issus du cadre théorique. C'est à partir de cette base de données que vont être reconstruites les TPC, et cela suivant deux approches.

\section{Première approche pour la reconstruction de TPC}

La première approche consiste à reconstruire à partir de chaque épisode interactionnel une ou plusieurs TPC. Cette reconstruction se fait en prenant en compte les mots clés affectés à l'épisode ainsi que le sens donné à l'action de l'enseignante au sein de cet épisode. Différentes façons de procéder ont été rendues explicites, en fonction de la catégorie de TPC à reconstruire (connaissances sur les difficultés, sur le curriculum etc.). Nous donnons quelques exemples de reconstruction pour différentes catégories de TPC:

\section{Exemple 1: TPC de la catégorie "difficultés"}

$$
\begin{aligned}
& \text { Locuteurs Productions verbales } \\
& \mathrm{E} \quad \mathrm{Il} \text { sert à quoi en fait heu K } \\
& \mathrm{P} \quad \text { Alors à quoi sert K/alors Taux on a compris à } \\
& \text { quoi ça servait } \mathrm{K} \text { bah en fait donc } \\
& \mathrm{K} \text { on a dit c'est indépendant/d'état initial ça veut dire } \\
& \text { si on a la transformation on connaît la valeur de K/et K } \\
& \text { ça relie les concentrations à l'état final/donc ça va nous } \\
& \text { permettre aussi de revenir à taux }
\end{aligned}
$$

Extrait 4: Transcription d'un épisode interactionnel mettant en avant une question d'élève qui comporte de l'implicite sur la difficulté éprouvée. 
La question de l'élève est générale, et peut être interprétée de différentes façons: dans quel but a t-on introduit cette constante d'équilibre (K) dans la classe, en quoi est-ce un concept scientifique utile?... La réponse de l'enseignante laisse comprendre qu'elle a interprété la question comme « comment va être utilisé $\mathrm{K}$ dans les exercices » puisqu'elle répond en expliquant à l'élève les situations où K sera utilisé: pour déterminer des concentrations et le taux d'avancement. L'enseignante a donc fait un choix à partir du sens attribué à la question de l'élève. C'est la présence d'un choix (qui apparaît pratiquement et donc sans nécessairement avoir été réfléchi comme tel, dans l'intervention de l'enseignante) qui nous permet de reconstruire une TPC. Cette TPC est formulée de la façon suivante: "Les élèves ont besoin de savoir à quoi va servir $\mathrm{K}$ dans les exercices ».

\section{Exemple 2: TPC de la catégorie "Curriculum "}

\section{Locuteur Productions verbales}

E madame est ce qu'on peut savoir si enfin les ions spectateurs ils nous le disent ou/

$\mathrm{P} \quad$ ah bah non ils le disent ça veut dire il faut que tu regardes heu par exemple dans un dosage conductimètrique si tu doses avec la soude il faut que tu penses que la soude c'est HO- qui va participer à la réaction de dosage

Extrait 5: Transcription d'un épisode interactionnel à partir duquel une TPC de la catégorie Curriculum à été reconstruite.

La TPC reconstruite ici est: « les élèves doivent savoir lister, lors d'une analyse par conductimètrie, tous les ions spectateurs. » Cette TPC à été reconstruite du fait que l'élève pose une question sur ce qu'il faut savoir (il ne s'agit pas d'une question portant sur une difficulté conceptuelle).

\section{Deuxième approche pour la reconstruction des TPC}

La deuxième approche pour reconstruire des TPC consiste, à partir de patterns de mots clés, à identifier des caractéristiques de l'action. À partir de ces caractéristiques de l'action une ou plusieurs TPC peuvent être reconstruites. Les patterns de mots clés sont issus de l'Analyse Statistique Implicative (ASI)
(Gras, Kuntz, \& Briand, 2001), qui met en évidence des liens d'implications (c'est-à-dire de la forme « $\mathrm{Si}$ A alors, en général, $B$ »). Ainsi l'analyse des mots clés par l'ASI a montré, par exemple, que si le savoir en jeu dans la réponse de l'enseignante appartient au niveau de modélisation des objets du monde visible, alors le savoir en jeu dans la question de l'élève appartient lui aussi au niveau de modélisation des objets du monde visible. Ces liens d'implications entre niveaux de modélisation dans la question de l'élève et la réponse de l'enseignante sont vérifiés pour pratiquement tous les niveaux de modélisation. À partir de l'ensemble de ces liens d'implication nous avons reconstruit la caractéristique de l'action suivante: le niveau de modélisation du savoir en jeu dans la réponse de l'enseignante est le même que dans la question de l'élève. De cette caractéristique de l'action de l'enseignante nous avons reconstruit la TPC suivante: l'enseignante ne change pas de niveau de modélisation dans sa réponse par rapport à celui de la question de l'élève. Il s'agit d'une TPC «stratégie».

\section{Mise en ouvre des TPC}

Afin d'étudier la mise en œuvre de ces TPC au cours d'un enseignement de chimie, nous explorons les liens d'implications entre la ou les catégories de TPC mises en œuvre dans l'épisode et les mots clés relatifs d'une part au type de savoir en jeu (niveau de modélisation) et d'autre part à la description de la mise en œuvre du savoir (chronogénèse, topogénèse.). Cette analyse statistique des épisodes interactionnels est accompagnée d'un retour vers les épisodes avec une approche narrative. Ainsi en ce qui concerne les TPC "Stratégies », par exemple, l'ASI montre que si l'enseignante fait un rappel, elle met en œuvre une TPC « Stratégies ». Le fait de faire un rappel pour répondre à une question d'élève est, de plus, pour l'enseignante, l'occasion d'utiliser des éléments du milieu mis en place précédemment. Ces éléments font partie du contexte dans lequel est construit le savoir par la classe. Pour l'enseignante faire un rappel est donc l'occasion de mettre en place une stratégie et d'utiliser des éléments du milieu. Ceci nous amène à plusieurs explications:

- Pour l'enseignante, faire un rappel nécessite la mise en place d'une stratégie: elle ne se contente pas de simplement répéter les choses. 
- Lors d'un rappel elle utilise des éléments du milieu mis en place précédemment, ce qui peut avoir un double but:

- Replacer les élèves dans le contexte

- La chimie étant une discipline où les représentations ont un rôle important, d'aider les élèves à suivre ce qu'elle dit.

Nous montrons un exemple qui permet d'illustrer ce qui vient d'être dit.

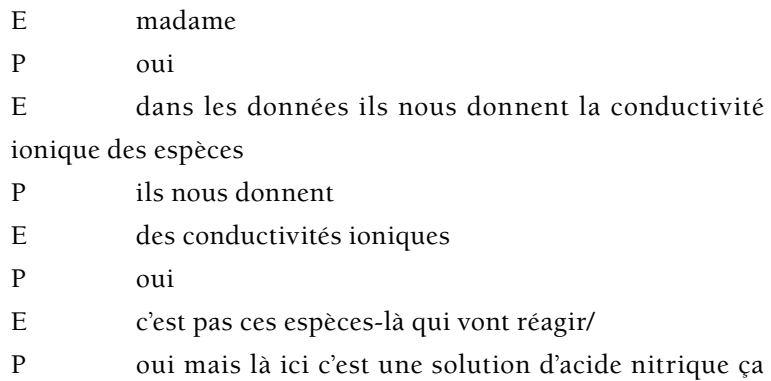
veut dire quoi quand on met dans l'eau l'acide il se dissocie donc il se dissocie comment en donnant $\mathrm{H}_{3} \mathrm{O}{ }^{+}$plus la base conjuguée mais quand on va chercher la concentration c'est la concentration en soluté apporté qu'on cherche d'accord alors on a dit quoi de la lumière/

\section{Extrait 6: Transcription d'un épisode interactionnel à partir} duquel une TPC "Stratégie » a été reconstruite.

Nous nous intéressons à la deuxième partie de la transcription (à partir de la question de l'élève: « c'est pas ces espèces-là qui vont réagir »), nous avons mis l'épisode interactionnel précédent pour pouvoir comprendre. La classe est en train de corriger un exercice.

Lenseignante demande aux élèves de chercher des valeurs de constantes concernant les espèces en jeu dans la réaction chimique. Un élève fait remarquer à l'enseignante que ces valeurs sont données (conductivités ioniques). Un autre élève demande à l'enseignante si les espèces pour lesquelles sont données ces constantes ne sont pas les espèces qui vont réagir.

L'enseignante lui répond que non parce que l'espèce pour laquelle la conductivité ionique est donnée est l'acide nitrique, alors que la classe étudie une solution d'acide nitrique, c'est-à-dire que l'acide nitrique a réagi avec l'eau pour donner des ions $\mathrm{H}_{3} \mathrm{O}^{+}$et la base conjuguée. Au tableau l'équation de la réaction entre l'acide nitrique et l'eau est écrite. L'enseignante

rappelle donc ce que la classe a vu précédemment: l'acide nitrique réagit avec l'eau. Elle pointe du doigt au tableau l'équation de la réaction et les représentants des espèces lorsqu'elle en parle. La TPC reconstruite est: «L'enseignante pointe du doigt les représentants des concepts auxquels elle fait allusion afin d'aider les élèves à comprendre en variant les représentants d'un même objet (langue naturelle et formule brute)».

En ce qui concerne les liens entre la catégorie de TPC « Stratégie » et le type de savoir en jeu, l'ASI nous montre que si l'enseignante fait un lien entre une grandeur et une propriété du monde non perceptible, alors elle met en œuvre une TPC «Stratégie». De plus si une TPC « Stratégie » est mise en cuvre lorsque l'enseignante fait un lien entre une grandeur et une propriété du monde non perceptible, alors la question de l'élève concerne un lien entre une grandeur et une propriété du monde non perceptible. Linterprétation est la suivante: lorsqu'il s'agit de faire ces liens entre grandeurs et propriétés l'enseignante dispose de stratégies lui permettant de répondre aux questions d'élèves. Ces connaissances sur les stratégies sont mises en œuvre lorsque la question de l'élève concerne le même niveau de modélisation que dans la réponse de l'enseignante. Il s'agit de quelque chose qui transparaît lors de la visualisation des extraits: les élèves sont souvent en quête de liens entre les grandeurs étudiées et des propriétés au niveau du monde non-perceptible. Les grandeurs étudiées dans cette séquence sont des grandeurs pour lesquelles les élèves ont du mal à donner une signification autre que mathématique (il s'agit de grandeurs thermodynamiques). L'enseignante se retrouve alors à devoir faire ces liens. Ces grandeurs sont souvent difficiles à interpréter au niveau non-perceptible (par exemple la constante de réaction est définie par son expression mathématique par l'enseignante, alors que les élèves cherchent à en avoir une interprétation au niveau perceptible ou non-perceptible). Pour faire ces liens difficiles l'enseignante dispose de stratégies (par exemple en comparant avec d'autres grandeurs).

Au travers de cette étude de la mise en œuvre des catégories de TPC en fonction du type de savoir en 
jeu, il s'agit de questionner les liens entre l'action, les connaissances professionnelles de l'enseignante et le savoir :

- L'action didactique, décrite au travers de la chrono- et de la topogenèse;

- Les connaissances professionnelles, exprimées sous forme de TPC;

- Le savoir, en termes de type de savoir.

\section{Résultats}

Dans cette partie nous présentons les résultats de notre analyse en termes de mise en œuvre des catégories de TPC. Dans un premier temps seront mis en évidence les liens entre connaissances professionnelles de l'enseignante et l'action didactique, puis les liens entre connaissances professionnelles de l'enseignante et le savoir enseigné en jeu.

\section{Liens entre connaissances professionnelles et action didactique}

Nous avons cherché à caractériser les TPC à l'aide des trois catégories de description de la mise en œuvre du savoir enseigné. L'analyse statistique implicative des mots clés relatifs à la mise en œuvre du savoir enseigné et les mots clés relatifs aux catégories de TPC ne montre pas de relation claire entre action didactique et catégories de TPC, sauf en ce qui concerne les catégories « l'enseignante fait un rappel (d'une autre classe) » et «l'enseignante introduit un savoir nouveau ». Pour expliquer cet état de fait il faut revenir sur les catégories de description du savoir enseigné et des TPC.

Les catégories de description de la mise en œuvre du savoir enseigné reviennent à décrire l'action de l'enseignante: "l'enseignante ne répond pas tout de suite à la question de l'élève ", "l'enseignante introduit un savoir nouveau », « l'enseignante introduit de nouveaux éléments dans le milieu », etc. Cependant pour décrire l'action de l'enseignante suivant les catégories suivantes: «l'enseignante fait un rappel », " l'enseignante fait un rappel d'une autre classe ", «l'enseignante introduit un savoir nouveau », « l'enseignante fait le lien entre un savoir nouveau et ancien », il nous a fallu construire des ensembles de savoirs. Si l'on considère le savoir en jeu dans un thème comme constituant un ensemble d'éléments de savoir, nous pouvons en effet définir différents ensembles de savoirs pour un thème donné: l'ensemble constitué par le savoir en jeu dans les thèmes précédents, l'ensemble constitué par le savoir en jeu dans les autres classes, l'ensemble constitué par le savoir qui n'est présent ni dans les thèmes précédents ni dans les autres classes (savoir nouveau). Ces ensembles de savoir nous permettent de situer le savoir en jeu dans l'épisode. Ainsi à la base de la description de l'action de l'enseignante en ce qui concerne les rappels, l'introduction d'un savoir nouveau etc. il y a une description de la dynamique $d u$ savoir. Les catégories de TPC sont construites autour de domaines de savoir professionnels, ces domaines sont les difficultés d'élèves, les stratégies l'enseignement, le curriculum et les buts et valeurs de l'enseignement des sciences. Il s'agit donc de catégoriser les connaissances professionnelles en fonction du domaine de savoir professionnel sur lequel ces connaissances professionnelles portent. Comme nous l'avons dit, l'analyse implicative ne montre pas de liens entre ces catégories de connaissances professionnelles (les catégories de TPC) et les catégories de description de l'action de l'enseignante (catégories relatives à la mise en œuvre du savoir enseigné). Ce résultat n'est pas surprenant: aucune de nos hypothèses ne permet de faire un lien entre une description de l'action relative à la mise en œuvre du savoir enseigné et une description des connaissances professionnelles basée sur des domaines de savoir. Il s'agit de deux dimensions de description de l'action de l'enseignante dans sa classe qui se situent à des niveaux différents. Il s'agit ici de deux " mises en œuvre » remplissant des fonctions propres et de deux types de savoirs distincts. Alors que le savoir enseigné est formulé par l'enseignante, les TPC ne sont pas explicites et sont sous-sous-jacente à l'action. Il existe par contre un lien entre les catégories « l'enseignante fait un rappel », " l'enseignante fait un rappel d'une autre classe » et "l'enseignante introduit un savoir nouveau » et les catégories de TPC.

Nous l'avons dit plus haut ces catégories de description de l'action reposent sur une différenciation du savoir en jeu dans l'épisode selon les ensembles de savoir présentés ci-dessus. Il s'agit 
d'une description du savoir enseigné. Il existe donc un lien entre la description du savoir enseigné et la description des connaissances professionnelles.

\section{Liens entre connaissances professionnelles de l'enseignante et savoir enseigné en jeu}

Nous nous demandons si certains savoirs appellent la mise en œuvre de certaines TPC ou si la mise en ouvre de certaines TPC appelle certains types de savoirs: À partir de nos analyses et des catégories qui sont les nôtres, les TPC « Curriculum » ne sont liées à aucun type de savoir en particulier. Il s'agit de la seule catégorie de TPC dans cette situation. Ce résultat montre que les questions des élèves concernant « ce qu'il faut savoir » portent sur des niveaux de modélisation variés.

Lorsque le savoir en jeu est du niveau des grandeurs, des TPC de la catégorie «Buts et valeurs » sont mis en œuvre. Ce résultat est explicable par le fait que l'enseignante fait le lien entre la valeur obtenue de manière théorique (c'est-à-dire par le calcul) et celle obtenue de manière expérimentale lorsqu'elle répond aux élèves à propos des grandeurs. Ces liens qu'elle fait entre théorique et expérimental sont des moments privilégiés pour reconstruire des TPC «Buts et valeurs ${ }^{2} »$. Nous donnons un exemple (extrait 7).

Les épisodes à partir desquels certaines TPC «Buts et valeurs» sont reconstruites sont des épisodes au cours desquels les élèves posent des questions sur ce qu'il faut savoir. Les élèves posent principalement des questions sur les valeurs des grandeurs qu'ils doivent savoir ou non. Nous avons codé le savoir en jeu dans ces épisodes comme appartenant au niveau des grandeurs.

E il nous sera donné le pKa

P le pKa des couples soit on vous les fait calculer par d'autres données soit on vous les donne donc vous avez pas à apprendre par cœur les pKa des couples

Extrait 7: Transcription d'un épisode à partir duquel une TPC « Buts et valeurs » à été reconstruite et dont le savoir en jeu appartient au niveau des grandeurs. La TPC reconstruite est « Lapprentissage de la chimie comporte une part d'apprentissage par cour de certaines valeurs alors que d'autres valeurs n'ont pas besoin d'être apprises par cour».

\author{
E mais K c'est la vraie valeur \\ $\mathrm{P} \quad$ ouais bah enfin c'est la valeur qu'on a mesurée qu'on \\ admet heu [...] à 25 degré
}

Extrait 8: Transcription d'un épisode interactionnel à partir duquel une TPC "Buts et valeurs " a été reconstruite et dont le savoir en jeu est du niveau des grandeurs. La TPC reconstruite est « Montrer comment marche la science fait partie de l'enseignement des sciences, et permet de mieux comprendre la science».

Lorsqu'une TPC «Difficultés » est mise en œuvre le savoir en jeu est théorique. Pour nous ce lien reflète le fait que l'enseignante ne possède que des connaissances relatives aux difficultés des élèves d'ordre théorique. Ce point soulève la question, que nous n'avons pas abordée dans ce travail, du développement des TPC chez les enseignants. Ces connaissances se développent en grande partie à travers l'expérience (van Dreil, 1998). L'enseignante observée étant encore relativement peu expérimentée (quatre ans d'enseignement dont deux en Terminale) cette explication semble vraisemblable.

Enfin lorsque le savoir en jeu concerne le lien entre une grandeur et une propriété du monde nonperceptible des TPC "Stratégies » sont mises en œuvre. Nous expliquons la mise en œuvre de TPC «Stratégies " lorsque le savoir en jeu concerne un tel lien par le fait que les grandeurs vues au cours de cette séquence d'enseignement sont des grandeurs thermodynamiques qui restent relativement abstraites. Le lien avec le monde non-perceptible ou perceptible n'est pas évident à faire, l'enseignante met ainsi en œuvre des stratégies pour aider les élèves.

Ainsi par exemple lorsqu'un élève demande à l'enseignante ce que représente le pKa, l'enseignante lui montre comment le pKa est relié au diagramme de prédominance. Le diagramme de prédominance représente les concentrations en espèces acides et basiques en fonction du $\mathrm{pH}$ pour une solution donnée. Le pKa correspond au point de $\mathrm{pH}$ pour lesquelles les concentrations en acide et en base sont identiques. En reliant le pKa, qui est une grandeur, au diagramme de prédominance, qui représente une propriété d'une solution (la concentration en fonction $\mathrm{du} \mathrm{pH}$ ) l'enseignante donne du sens à la grandeur pKa. Nous avons ainsi reconstruit la TPC suivante: «L'enseignante associe le pKa au diagramme de prédominance pour donner du sens au $\mathrm{pKa} »$. 
La caractérisation des TPC grâce au niveau de modélisation s'avère être riche puisqu'elle nous permet de mettre en relief les spécificités des connaissances de l'enseignante. Elle nous a également permis de caractériser les questions d'élèves concernant ce qu'il faut savoir.

Ces deux analyses, catégories de connaissances professionnelles en fonction du savoir en jeu et en fonction de la mise en œuvre du savoir, nous permettent ainsi de conclure que les catégories de TPC sont liées au savoir mais pas à sa mise en œuvre. Cela ne veut pas dire qu'il n'existe pas des cas où une TPC est mise en œuvre en fonction de la mise en œuvre du savoir, mais qu'il n'existe pas de lien d'implication (dans un sens ou l'autre) entre catégorie de TPC et catégorie de description de la mise en œuvre du savoir dans la classe et donc de l'action didactique.

\section{Conclusion}

Nous l'avons dit, les PCK sont une approche heuristique intéressante en ce qui concerne la formation des enseignants, mais ce cadrage est peu théorisé et surtout il est peu spécifié en fonction des savoirs. Notre cadre théorique propose d'importer les catégories de connaissances construites avec l'approche en termes de PCK dans le cadre de la TACD. Notre approche, qui part de l'action du professeur en classe, permet de dépasser le plan du dicible, et ainsi met en lumière des connaissances mises en œuvre par les enseignants sans qu'ils en aient forcément conscience. Nous avons pour cela mis au point une méthode de reconstitution d'ensembles de connaissances mises en œuvre dans des situations de classe bien précises, les épisodes interactionnels, et ce à partir de l'analyse en termes de chronogenèse et topogenèse de l'action didactique. Cette méthode, basée sur des mots clés et une analyse statistique, permet de mettre en évidence des régularités ou patterns, dans l'action du professeur. Cette méthode impose de limiter les catégories de descripteurs de l'action pour pouvoir faire émerger des patterns. Ceux-ci sont à discuter en fonction de leur cohérence par rapport à notre cadre théorique.

Notre approche permet également de pouvoir dépasser le simple catalogue de connaissances en liant ces connaissances avec des pratiques de classe. Cette possibilité est rendue accessible grâce à l'aller retour entre une analyse «narrative » et une analyse statistique au cœur de notre méthode d'analyse. En effet l'analyse statistique donne des patterns de mots clés, mis en lumière à partir de plusieurs extraits vidéo, alors que l'analyse " discursive » permet de donner un sens à ces patterns en prenant en compte les spécificités de la situation. Il est alors possible de distinguer le générique du spécifique, et donc de rendre compte de connaissances mises en jeu non seulement du point de vue de la spécificité de la situation mais aussi d'aspects plus génériques mis en lumière grâce à l'utilisation des mots clés pour caractériser les épisodes.

Le système de catégories de connaissances construites par l'approche en termes de PCK porte la trace des hypothèses qui ont conduit à sa construction. Ainsi, les catégories mises à jour par les différents travaux sont centrées sur les connaissances reconstruites à partir de ce que les enseignants disent de leur pratique. Au vu du nombre d'études portant sur les PCK, les catégories en questions sont vraisemblablement pertinentes lorsqu'il s'agit d'étudier ces connaissances. Afin de dépasser le problème posé par le lien entre ce que les enseignants disent de leur pratique et ce qu'ils font dans leur pratique nous abordons l'étude des connaissances professionnelles par l'étude de l'action didactique. Ces connaissances professionnelles ne sont plus reconstruites à partir d'un discours ou d'un questionnaire, mais à partir de l'action, elles changent ainsi de statut et deviennent un émergeant de l'action étudiée au travers de l'action didactique et dans le cadre de celle-ci. Notre étude montre que certaines des catégories de connaissances construites dans le cadre des PCK restent pertinentes pour catégoriser les connaissances construites avec notre approche. Ainsi nous avons pu recourir aux catégories " connaissances sur les difficultés des élèves » et « connaissances sur le curriculum » pour différencier certaines des connaissances reconstruites. Nous faisons l'hypothèse que la catégorie « connaissances sur l'évaluation » est également susceptible de garder sa pertinence. Par contre les catégories « Buts et valeurs " ainsi que " connaissances sur les stratégies d'enseignement $»$ sont plus problématiques. Les seules connaissances reconstruites qui correspondent à la catégorie «Buts et valeurs» sont des connaissances liées à l'épistémologie de l'enseignante (ses connaissances relatives au fonctionnement de la chimie). Nous n'avons pu reconstruire de TPC qui 
s'expriment sous forme d'un but ou d'une valeur de l'enseignante. Ceci serait dû au fait qu'il n'existe peu (voire pas?) d'observables dans l'action pour ce type de connaissance. Ainsi l'inférence qu'il est nécessaire de faire pour reconstruire, à partir de notre analyse de l'action, des buts ou des valeurs de l'enseignant est trop important. La catégorie " connaissance sur les stratégies d'enseignement " pose également problème, car la définition de ce qu'est une stratégie d'enseignement est loin d'être claire. Ainsi les TPC que nous avons reconstruites et qui appartiennent à cette catégorie sont plus une description d'une action effective qu'une connaissance Lorsqu'il s'agit d'étudier des connaissances à partir de ce que les enseignants disent de leur pratique, il semble raisonnable de regrouper dans une même catégorie toutes les connaissances relatives à ce que les enseignants "font en vue de ». Si l'on reprend la définition donnée dans la partie théorique sur les PCK il s'agit des connaissances professionnelles exprimées en vue d'atteindre un but, d'aider les élèves à dépasser une ou des difficultés, de présenter le savoir à enseigner d'une manière adéquate... il s'agit également des connaissances sur les analogies, sur les métaphores, sur les types de situations. Le problème semble être lié au changement de perspective que nous proposons pour étudier les connaissances professionnelles. En passant de l'étude de ce que les enseignants disent de leur pratique pour reconstruire des connaissances professionnelles à l'étude de leur pratique pour reconstruire des connaissances professionnelles, la catégorie « connaissances sur les stratégies d'enseignement » qui regroupe, dans l'approche PCK les connaissances reconstruites à partir d'un discours de ce que les enseignant " font " perd sa consistance. En effet comment sélectionner, lorsque l'on étudie l'action, telle ou telle action représentative d'une stratégie? Est ce que l'ensemble des actions de l'enseignant dans sa classe ne constitue pas une stratégie ou une suite imbriquée des stratégies? En adoptant une approche « actionnel » les connaissances reconstruites ne sont plus du type « connaissances sur » mais « connaissances dans ». Ce changement de perspective affecte différemment les catégories de TPC: alors que les connaissances de type « difficultés d'élèves » ou « connaissances sur le curriculum» semblent pouvoir être traitées de la même façon dans leur relation à l'action, lorsqu'il s'agit de connaissances sur l'action et de connaissances dans l'action, la catégorie «Stratégies d'enseignement » nécessite une reconfiguration.

Une manière de considérer les TPC relevant d'une stratégie d'enseignement pourrait être de définir celle-ci comme le lien ou la relation que fait l'enseignant entre TPC de différentes catégories. Par exemple le fait pour un enseignant de poser une question à un élève afin d'évaluer sa compréhension d'un concept pourrait être interprété comme la mise en relation d'une TPC « évaluation» (comment évaluer, quelle question poser) et une TPC « difficulté d'élève » (quelle difficulté est ciblée ou détectée par l'enseignant). Cette mise en relation, sur laquelle le modèle des PCK insiste, serait ainsi conceptualisée comme un type de connaissance dans l'action relatif à la mise en place d'une "stratégie». 


\section{NOTES}

1. Nous suivons la distinction proposée par Brousseau (http://pagesperso-orange.fr/daest/guy-brousseau/ textes/Glossaire_Brousseau.pdf) concernant la distinction entre savoirs et connaissances.

2. Le mot valeur a deux significations distincts: concept décrivant les croyances et les convictions d'un individu dans le cas de la catégorie de TPC «Buts et Valeurs », et produit d'un nombre et d'une unité physique dans le cas de la valeur d'une grandeur.

\section{RÉFÉRENCES}

Abell, S. K. (2007). Research on science teacher knowledge. In S. K. Abell \& N. G. Lederman (Eds.), Handbook of research on science education (p. 1105-1149). Mahwa, New Jersey: Lawrence Erlbaum Associates.

Brousseau, G. (1998). Théorie des situations didactiques. Grenoble: La pensée sauvage.

Buty, C., Tiberghien, A., \& Le Maréchal, J. F. (2004). Learning hypotheses and associated tools to design and to analyse teaching-leaning sequences. International Journal of Science Education, 26 (5), (p. 579-604).

Carter, K. (1993). The place of story in the study of teaching and teacher education. Educational Research, 22, (p. 5-12).

Gras, R., Kuntz, P., \& Briand, H. (2001). Les fondements de l'analyse statistique implicative et quelques prolongements pour la fouille de données. Mathématiques et Sciences Humaines, 154, (p. 9-29). Retrieved from http://msh.revues.org/document2849.html

Hashweh, M. Z. (2005). Teacher pedagogical constructions: a reconfiguration of pedagogical content knowledge. Teachers and Teaching: theory and practice. 11 (3), (p. 273-292).

Kagan, D. M. (1992). Professional Growth Among Preservice and Beginning Teacher. Review of Educational Research, 62 (2), (p. 129-169).

Le Maréchal, J.-F., \& Bécu-Robinault, K. (2006). La simulation au sein du projet Microméga. Aster43, (p. 81-108).

Le Maréchal, J. F. (1999). Modelling student's cognitive activity during the resolution of problems based on experimental facts in chemical education. In J. Leach \& A. C. Paulsen (Eds.), Practical work in science education. Dordrecht: Kluwer Academic Publisher. (p. 195-209).

Magnusson, S., Krajcik, J., \& Borko, H. (1999). Nature, sources, and development of pedagogical content knowledge for science teaching. In N. G. L. Julie Gess-Newsome (Ed.), Examining Pedagogical Content Knowledge (p. 95 - 132). Boston: Kluwer.

Martinand, J.-L. (1992). Enseignement et apprentissage de la modélisation. Paris: INRP.

Park, S., Oliver, J.-S. (2008). Revisiting the Conceptualisation of Pedagogical Content Knowledge (PCK): PCK as a Conceptual Tool to Understand Teachers as Professionals. Research in Science Education. 38 (3), 261-284. 
Rollnick, M., Bennett, J., Rhemtula, M., Dharsey, N., \& Ndlovu, T. (2008). The Place of Subject Matter Knowledge in Pedagogical Content Knowledge: A Case Study of South African Teachers Teaching the Amount of Substance and Chemical Equilibrium. International Journal of Science Education, 30 (10), (p. 1365-1387).

Schubauer-Leoni, M-L., Leutenegger, F., Ligozat, F.\& Fluckiger, A. (2007). Un modèle de l'action conjointe professeur-élèves: Les phénomènes didactiques qu'il peut/doit traiter. In G. Sensevy \& A. Mercier (Eds.), Agir ensemble: Éléments de théorisation de l'action conjointe du professeur et des élèves. (p. 51-91). Rennes: PUR.

Sensevy, G. (2007). Des catégories pour décrire et comprendre l'action didactique. In G. Sensevy \& A. Mercier (Eds.), Agir ensemble: Éléments de théorisation de l'action conjointe du professeur et des élèves (p. 13-49). Rennes: PUR.

Shulman, L. S. (1986). Those who understand: knowledge growth in teaching. Educational Researcher, 15 (2), (p. 4-14).

Shulman, L. S. (1987). Knowledge and teaching: Foundation of a new reform. Harvard Education Review, 57 (1), (p. 1-21).

Tiberghien, A. (1994). Modelling as a basis for analyzing teaching-learning situations. Learning and Instruction, 4 (1), (p. 71-87).

Tiberghien, A. (2000). Designing teaching situations in the secondary school. In R. Millar, J. Leach \& J. Osborne (Eds.), Improving science education: The contribution of research (p. 27-47). Buckingham, UK: Open University Press.

Tiberghien, A., Malkoun, L., Buty, C., Souassy, N., \& Mortimer, E. (2007). Analyse des savoirs en jeu en classe de physique à différentes échelles de temps. In G. Sensevy \& A. Mercier (Eds.), Agir ensemble: Éléments de théorisation de l'action conjointe du professeur et des élèves. (p. 93-122). Rennes: PUR.

van Driel, J. H., Verloop, N., \& de Vos, W. (1998). Developing Science Teachers'Pedagogical Content Knowledge. Journal of Research in Science Teaching, 35 (6), (p. 673-695). 